\title{
Arando na profilaxia das infecções urinárias recorrentes: revisão baseada na evidência
}

Alexandra Pina, ${ }^{1}$ Ana Raquel Figueiredo, ${ }^{1}$ Ana Campos, ${ }^{2}$ Clara Pinto Ferreira, ${ }^{1}$ Irene Lopes, ${ }^{3}$ Nuno Figueiras Alves, ${ }^{4}$ Isabel Ribeiro ${ }^{5}$

\section{RESUMO}

Introdução: As infecções urinárias recorrentes têm um grande impacto em termos de morbilidade e custos em saúde. A emergente resistência aos antibióticos acentua a necessidade de terapêuticas profilácticas alternativas.

Objectivo: Rever a evidência disponível sobre a eficácia do arando na profilaxia das infecções não complicadas do tracto urinário em mulheres adultas.

Fontes de dados: Base de dados MEDLINE e sítios de Medicina Baseada na Evidência (UpToDate, Clinical Evidence, Bandolier, EBM Online, ACP Journal Club, TRIP Database, InfoPOEMs, The Cochrane Library, DARE, Guidelines Finder, National Guideline Clearinghouse, Canadian Medical Association Infobase, USPSTF, NICE e Canadian Task Force on Preventive Health Care).

Métodos de revisão: Pesquisa de sistemas, meta-análises, revisões sistemáticas, normas de orientação clínica e ensaios clínicos aleatorizados e controlados, publicados entre Janeiro de 2000 e Dezembro de 2010, em inglês, português e espanhol. Foram utilizados os termos MeSH, vaccinium macrocarpon e urinary tract infections, e a palavra cranberry. Para avaliação da qualidade dos estudos e força de recomendação foi utilizada a escala Strength of Recommendation Taxonomy da American Family Physician.

Resultados: Foram obtidos 127 artigos, dos quais nove cumpriam os critérios de inclusão: um sistema, três revisões sistemáticas, quatro normas de orientação clínica e um ensaio clínico. O arando, nas suas diferentes formas, parece ser eficaz na redução da recorrência das infecções urinárias em mulheres adultas sem patologias associadas.

Conclusões: A evidência disponível demonstrou que o arando apresenta benefício na profilaxia das infecções urinárias recorrentes (Força de Recomendação B). Contudo, a sua recomendação está limitada pela heterogeneidade do desenho dos estudos e pela falta de consenso relativamente à dosagem e formulação a utilizar. São, por isso, necessários estudos de qualidade, que avaliem a segurança e tolerabilidade, bem como a posologia e a formulação mais adequadas.

Palavras-chave: Arando; Infecções do tracto urinário; Vaccinium macrocarpon.

\section{INTRODUÇÃO}

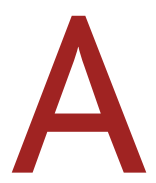
s infecções do tracto urinário (ITU) recorrentes na mulher têm um grande impacto em termos de morbilidade e custos em saúde, pelo que é fundamental a sua prevenção. ${ }^{1}$ As cistites agudas não complicadas são frequentes em mulheres adultas e aproximadamente um terço destas

\footnotetext{
${ }^{1}$ Interna de Medicina Geral e Familiar na Unidade de Saúde Familiar Horizonte, ACES de Matosinhos.

${ }^{2}$ Interna de Medicina Geral e Familiar na Unidade de Saúde Familiar Leça, ACES de Matosinhos.

${ }^{3}$ Interna de Medicina Geral e Familiar na Unidade de Saúde Familiar Oceanos, ACES de Matosinhos.

${ }^{4}$ Médico de Família na Unidade de Cuidados de Saúde Personalizados de Matosinhos, ACES de Matosinhos.

IInterna de Medicina Geral e Familiar na Unidade de Cuidados de Saúde Personalizados de Matosinhos, ACES de Matosinhos.
}

apresenta ITU recorrentes, definidas como a existência de, pelo menos, duas infecções durante seis meses ou três episódios durante um ano. ${ }^{2}$

Diferentes abordagens têm sido propostas para a prevenção das ITU recorrentes, incluindo antibioterapia profiláctica e terapêuticas não farmacológicas ( $L a c$ tobacillus, probióticos, vacinas e estrogénios intravaginais). ${ }^{2}$ A emergente resistência aos antibióticos acentua a necessidade de terapêuticas profilácticas alternativas, contextualizando o interesse crescente pelo arando (cranberry, vaccinium macrocarpon). Trata-se de um fruto vermelho abundante na costa leste dos Estados Unidos da América, usado com fins medicinais desde o século XVII e objecto de estudo, desde há várias décadas, na prevenção da recorrência das ITU. ${ }^{1}$ Em Por- 
tugal, estão disponíveis vários produtos que incluem o arando na sua composição.

O arando é composto por água, ácidos orgânicos, glicose, vitamina C, frutose e um tipo específico de flavonóides, as proantocianidinas, um subtipo de taninos. Actualmente, considera-se que o seu mecanismo de acção na profilaxia das ITU se deve à capacidade das proantocianidinas e da frutose inibirem a adesão das fímbrias dos uropatogénios (principalmente da Escherichia coli) às células uroepiteliais e, assim, impedirem a colonização e o crescimento bacteriano. ${ }^{3-4}$

O objectivo deste estudo é rever a evidência disponível sobre a eficácia do arando na profilaxia da recorrência das ITU em mulheres adultas saudáveis.

\section{MÉTODOS}

Realizou-se uma pesquisa nas bases de dados $M E$ DLINE, UpToDate, Clinical Evidence, Bandolier, EBM Online, ACP Journal Club, TRIP Database, InfoPOEMs, The Cochrane Library, DARE, Guidelines Finder, National Guideline Clearinghouse, Canadian Medical Association Infobase, USPSTF, NICE e Canadian Task Force on Preventive Health Care, de sistemas, meta-análises, revisões sistemáticas, normas de orientação clínica e ensaios clínicos aleatorizados e controlados, publicados entre Janeiro de 2000 e Dezembro de 2010, nas línguas inglesa, portuguesa e espanhola. Foram utilizados os termos MeSH, Vaccinium macrocarpone Urinary tract infections, e a palavra Cranberry.

Foram utilizados os seguintes critérios de inclusão:
- População: mulheres saudáveis, com idade igual ou superior a 18 anos, com ITU recorrentes;

- Intervenção: arando (nas diferentes formulações disponíveis);

- Comparação: placebo ou outros métodos profilácticos de ITU;

- Outcome: diminuição do número de episódios de ITU. Foram excluídas mulheres com factores de risco para infecções urinárias recorrentes mais frequentes ou complicadas, como gravidez, Diabetes Mellitus, imunossupressão, anomalia anatómica ou funcional do tracto urinário, algaliação, infecção urinária activa e hospitalização, manipulação do tracto urinário ou antibioterapia recentes.

Para avaliação da qualidade dos estudos e atribuição de força de recomendação (FR) foi utilizada a escala Strength of Recommendation Taxonomy da American Family Physician (Quadro I). ${ }^{5}$

\section{RESULTADOS}

Com a pesquisa inicial foram identificados 127 artigos, dos quais nove cumpriam os critérios de inclusão: um sistema, ${ }^{6}$ três revisões sistemáticas, ${ }^{7-9}$ um ensaio clínico ${ }^{10}$ e quatro normas de orientação clínica. ${ }^{11-14}$

Na revisão do Uptodate, de 2009, sobre infecções recorrentes do tracto urinário, ${ }^{6} \mathrm{o}$ sumo de arando é referido como sendo uma estratégia profiláctica eficaz, embora a sua recomendação seja limitada pela inconsistência quanto à duração da terapêutica e às diferentes formulações e doses de arando utilizadas nos vários es-

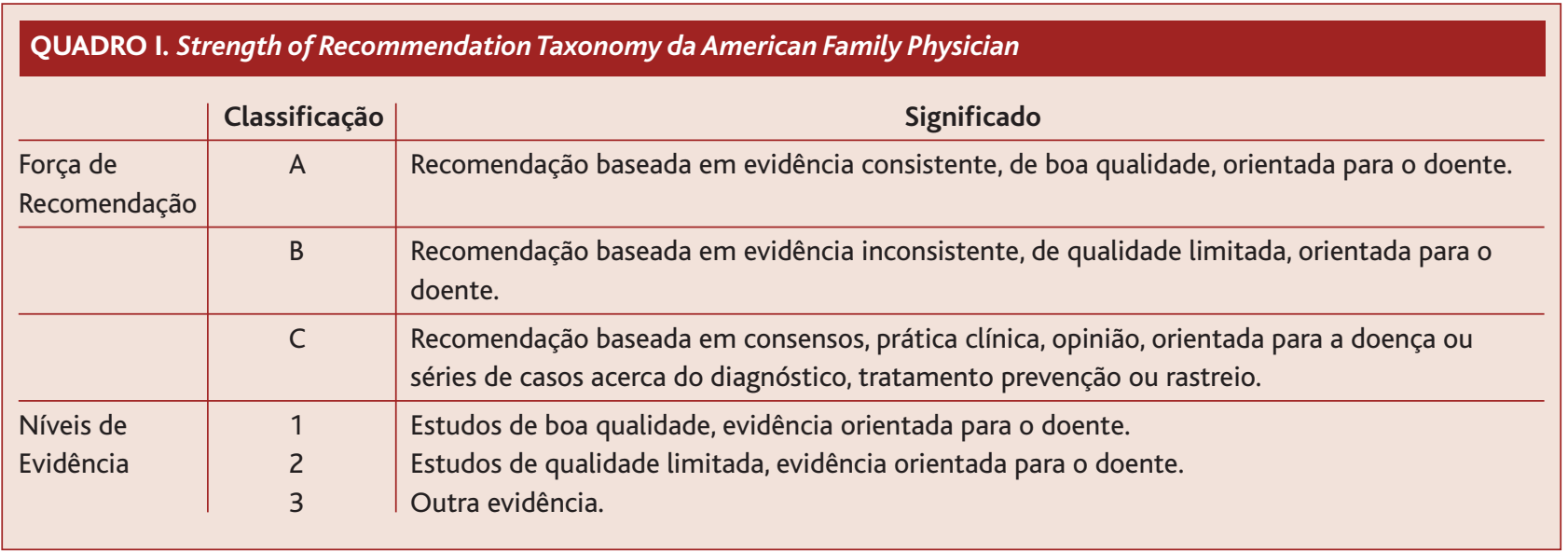


tudos, pelo que lhe foi atribuída uma Força de Recomendação B (Quadro II).

As três revisões sistemáticas foram classificadas com um Nível de Evidência 2 por levantarem questões relativamente às disparidades metodológicas dos vários estudos analisados. No entanto, todas afirmaram que existe evidência a favor da redução da taxa de recorrências/ano com a utilização de compostos de arando na prevenção de ITU recorrentes em comparação com placebo ou Lactobacillus (Quadro III). ${ }^{7-9}$

Segundo a revisão da Bandolier, de 2007, que incluiu quatro estudos com um total de 779 participantes (85\% mulheres, com média de idades de 58 anos), o arando (sumo ou comprimidos) mostrou efeito na prevenção da recorrência de ITU (RR=0,5 IC 95\%; 0,40-0,80; NNT=14). A duração dos ensaios analisados variava entre seis e doze meses e as doses de arando entre 50 e $350 \mathrm{ml}$ de sumo ou comprimidos com 1:30 partes de sumo concentrado. Porém, nenhum dos estudos justificou o motivo pelo qual foram definidas as dosagens utilizadas. A posologia variou entre toma diária única ou múltiplas tomas (Quadro III).?

A revisão da Cochrane, de 2008, incluiu 10 estudos e um total de 1049 participantes (mulheres entre os $21 \mathrm{e}$ os 72 anos), utilizando doses e duração de tratamento semelhantes às analisadas pela Bandolier, e avaliou a eficácia do sumo ou comprimidos de arando. Destes

\section{QUADRO II. Sistema}

\begin{tabular}{|c|c|c|c|}
\hline Entidade e Ano & Conclusões & Limitações & FR \\
\hline UpToDate 2009 & $\begin{array}{l}\text { O sumo de arando é eficaz na prevenção das ITU } \\
\text { recorrentes. }\end{array}$ & $\begin{array}{l}\text { Uso de diferentes doses e formulações de arando. } \\
\text { Inconsistência quanto à duração da terapêutica. }\end{array}$ & B \\
\hline
\end{tabular}

FR: Força de Recomendação. ITU: Infecção tracto urinário.

\begin{tabular}{|c|c|c|c|c|c|}
\hline Referência & Estudos & Intervenção & Conclusões & Comentários & NE \\
\hline $\begin{array}{l}\text { Bandolier } \\
2007\end{array}$ & $\begin{array}{l}4 \text { ECAC } \\
(n=779)\end{array}$ & $\begin{array}{l}\text { Arando (sumo, comp) vs } \\
\text { Placebo/bebidas com } \\
\text { Lactobacillus. } \\
\text { Duração: } 12 \text { meses. }\end{array}$ & $\begin{array}{l}\text { A toma diária de sumo de } \\
\text { arando durante } 6 \text { a } 12 \text { meses } \\
\text { previne a recorrência de ITU } \\
\text { em uma em cada dez mulheres. } \\
\text { RR=0.5 } \\
\text { (IC } 95 \% ; 0.40-0.80) \text {. } \\
\text { NNT }=14 \text {. }\end{array}$ & $\begin{array}{l}\text { Heterogeneidades } \\
\text { metodológicas. }\end{array}$ & 2 \\
\hline $\begin{array}{l}\text { Jepson RG } \\
\text { Craig JC } \\
\text { (Cochrane) } \\
2008\end{array}$ & $\begin{array}{l}10 \text { estudos } \\
(n=1049)\end{array}$ & $\begin{array}{l}\text { Arando (sumo, cáps.) vs } \\
\text { Placebo/sumo/água. }\end{array}$ & $\begin{array}{l}\text { O sumo de arando reduz } \\
\text { significativamente a incidência } \\
\text { de ITU aos } 12 \text { meses. } \\
\text { RR=0.65. } \\
\text { (IC } 95 \% ; 0.46-0.90) \text {. } \\
\text { NNT=31. }\end{array}$ & $\begin{array}{l}\text { Elevado número de } \\
\text { desistências (20-50\%) por } \\
\text { intolerância ao arando em } \\
\text { alguns estudos. } \\
\text { Dose e formulação ideais não } \\
\text { estabelecidas. }\end{array}$ & 2 \\
\hline $\begin{array}{l}\text { Guay } \\
2009\end{array}$ & $\begin{array}{l}4 \text { estudos } \\
(n=330)\end{array}$ & $\begin{array}{l}\text { Arando (sumo/caps.) vs } \\
\text { Placebo/Lactobacillus. } \\
\text { Duração: } 12 \text { semanas a } 12 \\
\text { meses. }\end{array}$ & $\begin{array}{l}\text { Não recomendado por falta de } \\
\text { consenso relativamente à } \\
\text { dosagem e formulação. } \\
\text { Redução de } 35 \% \text { na taxa de } \\
\text { recorrências/ano. NNT=3. }\end{array}$ & $\begin{array}{l}\text { Limitações na aleatorização e } \\
\text { na avaliação da adesão à } \\
\text { terapêutica. } \\
\text { Altas taxas de desistência. }\end{array}$ & 2 \\
\hline
\end{tabular}

NE: Nível de Evidência. ECAC: Ensaio clínico aleatorizado e controlado. ITU: Infecção tracto urinário. Vs: versus. RR: risco relativo. IC: intervalo de confiança. n: número amostral. Comp: comprimidos. Cáps: cápsulas. 
ensaios, apenas dois apresentavam características populacionais que correspondiam às elegíveis para a presente revisão. A meta-análise destes estudos permitiu encontrar um RR de 0,61 (IC 95\%; 0,40-0,91) associado ao uso de arando em comparação com placebo ou bebidas contendo Lactobacillus (NNT=31). Não se verificou diferença entre as formulações constituídas por sumo ou comprimidos. Concluiu-se haver uma redução significativa da incidência de ITU após 12 meses de tratamento com arando (Quadro III). ${ }^{8}$

A revisão sistemática de quatro ensaios, elaborada por Guay em 2009, engloba uma amostra de $330 \mathrm{mu}$ lheres com idades entre os 21 e os 75 anos. Para além das formulações referidas nas outras revisões sistemáticas, incluiu um estudo com a posologia de duas tomas diárias de comprimidos com 200 mg durante três meses. Esta revisão concluiu que o arando está associado a uma redução de $35 \%$ na taxa de recorrências ao ano (NNT=3). Contudo, não é recomendada a utilização do arando por falta de consenso relativamente à dosagem e formulação preconizadas e algumas limitações metodológicas dos estudos (Quadro III). ${ }^{9}$

O ensaio clínico de Mc Murdo et al., de 2009, foi o único estudo dos analisados que comparou a utilização do arando com a antibioterapia profiláctica. Este estudo incluiu 137 mulheres, com idade igual ou superior a 45 anos (média de 63 anos) e história de duas ou mais ITU recorrentes nos últimos 12 meses. A população do estudo foi dividida em dois grupos, um submetido a tratamento com $500 \mathrm{mg}$ de arando e outro submetido a trimetoprim $100 \mathrm{mg}$, um vez por dia, durante 6 meses ou até ao pri- meiro episódio de ITU. Verificou-se recorrência da ITU em $36,2 \%$ das doentes submetidas ao tratamento com arando, em comparação com $20,6 \%$ de recorrência no grupo do trimetoprim, sendo esta diferença não estatisticamente significativa $(\mathrm{p}=0,084)$. Não se registou igualmente diferença significativa no tempo para a primeira recorrência. Este estudo concluiu que o arando poderá ser menos eficaz do que o trimetoprim na redução da incidência de ITU sintomáticas. Tratou-se de um ensaio clínico com algumas limitações metodológicas, nomeadamente sem dupla ocultação descrita, pelo que foi classificado com Nível de Evidência 2 (Quadro IV). ${ }^{10}$

As forças de recomendação atribuídas pelas quatro normas de orientação clínica analisadas foram convertidas para a taxonomia SOR. ${ }^{5}$ As normas mais antigas, da Society of Obstetricians and Gynaecologists of Cana$d a$, de 2004, ${ }^{11}$ e da Scottish Intercollegiate Guidelines Network, de 2006, ${ }^{12}$ foram classificadas com Força de Recomendação A. A conversão da classificação das normas mais recentes, da EBM Guidelines, em 2009, ${ }^{13} \mathrm{e}$ da European Association of Urology, em 2010, correspondeu a Força de Recomendação B (Quadro V). ${ }^{14}$

A Society of Obstetricians and Gynaecologists of $\mathrm{Ca}$ nada refere que as mulheres com ITU recorrentes devem ser informadas de que o consumo de sumo de arando puro diminui o risco de ITU, em oposição às bebidas contendo arando na sua composição (Quadro V). ${ }^{11}$

A norma baseada na evidência publicada pela Scottish Intercollegiate Guidelines Network recomenda que mulheres adultas com infecções recorrentes do tracto urinário sejam aconselhadas a ingerir suplementos de

\section{QUADRO IV. Ensaio Clínico}

\begin{tabular}{|c|c|c|c|c|c|}
\hline Referência & $\mathbf{n}$ & Intervenção & Conclusões & Comentários & NE \\
\hline $\begin{array}{l}\text { Mc Murdo et al. } \\
2009\end{array}$ & $\begin{array}{l}\mathrm{n}=137 . \\
\text { Mulheres } \geq 45 \text { anos } \\
\text { (média } 63 \text { anos). }\end{array}$ & $\begin{array}{l}\text { Arando } 500 \mathrm{mg} \text { vs } \\
\text { Trimetoprim } 100 \mathrm{mg} \\
\text { 1x/dia. } \\
\text { Duração: } 6 \text { meses ou até } \\
\text { um novo episódio de ITU. }\end{array}$ & $\begin{array}{l}\text { Não há diferenças } \\
\text { estatisticamente } \\
\text { significativas entre o } \\
\text { arando e o trimetoprim na } \\
\text { prevenção das ITU } \\
\text { recorrentes. } \\
\text { RR=1.616. } \\
\text { (IC 95\%: } 0,93-2,79) \text {. }\end{array}$ & $\begin{array}{l}\text { Sem diferenças } \\
\text { significativas no tempo } \\
\text { até à primeira recorrência. }\end{array}$ & 2 \\
\hline
\end{tabular}

n: número amostral. NE: Nível de Evidência. ITU: Infecção tracto urinário. RR: risco relativo. IC: intervalo de confiança. 


\begin{tabular}{|c|c|c|}
\hline Referência & Conclusões & FR \\
\hline $\begin{array}{l}\text { Society of Obstetricians and } \\
\text { Gynaecologists of Canada } \\
2004\end{array}$ & $\begin{array}{l}\text { Mulheres com ITU recorrentes devem ser informadas de que o consumo de sumo de arando } \\
\text { puro diminui o risco de ITU. }\end{array}$ & A \\
\hline $\begin{array}{l}\text { Scottish Intercollegiate } \\
\text { Guidelines Network } \\
2006\end{array}$ & $\begin{array}{l}\text { Mulheres adultas com ITU recorrentes devem ser aconselhadas a tomar suplementos de } \\
\text { arando, para diminuir a frequência das recorrências. }\end{array}$ & $A$ \\
\hline EBM Guidelines 2009 & $\begin{array}{l}\text { O sumo de arando pode reduzir o número de ITU sintomáticas ao longo de um período de } 12 \\
\text { meses. }\end{array}$ & B \\
\hline $\begin{array}{l}\text { European Association of } \\
\text { Urology } 2010\end{array}$ & $\begin{array}{l}\text { O sumo de arando é um método profiláctico alternativo nas ITU recorrentes não complicadas } \\
\text { em mulheres jovens. }\end{array}$ & B \\
\hline
\end{tabular}

FR: Força de Recomendação. ITU: Infecção tracto urinário.

arando, de modo a diminuir a frequência das recorrências. Os autores referem ainda que mulheres hipocoaguladas com varfarina deverão evitar ingerir arando, a não ser que os benefícios sejam superiores aos riscos, com uma Força de Recomendação B (Quadro V). ${ }^{12}$

A EBM Guidelines, de 2009, afirma que o sumo de arando pode reduzir o número de ITU sintomáticas ao longo de um período de 12 meses, justificando esta recomendação essencialmente com base nas conclusões da Cochrane de 2008 (Quadro V). ${ }^{13}$

A European Association of Urology actualizou, em 2010, a sua norma de orientação clínica baseada na evidência, onde reforça a possibilidade de utilizar métodos alternativos aos antibióticos, como imunoterapia com lisados bacterianos, probióticos, acidificação urinária e suplementação com arando. Os autores consideram, pela análise dos artigos em que se baseiam, que o número de infecções do tracto urinário pode ser diminuído com o consumo de sumo de arando (Força de Recomendação B) na dose mínima de $36 \mathrm{mg} /$ dia de proantocianidinas. Ainda assim, esta alternativa parece não ser tão eficaz quanto os antibióticos profiláticos, embora não tenham sido analisado estudos que comparassem directamente estas duas abordagens (Quadro V). ${ }^{14}$

\section{CONCLUSÕES}

O arando mostrou-se eficaz na redução da recorrência das ITU em mulheres adultas sem patologias associadas (Força de Recomendação B). Contudo, a evidên- cia científica que suporta a eficácia do arando não é suficientemente robusta. Para isso contribuem algumas limitações da qualidade dos estudos, pois apresentam amostras reduzidas com elevadas taxas de desistência. Paralelamente, existe alguma heterogeneidade no desenho dos estudos, com utilização de dosagens díspares e diferentes vias de administração ou formulações.

O potencial impacto desta recomendação na prática clínica torna premente a realização de novos estudos, desenhados com metodologias mais correctas e incluindo um maior número amostral, que comparem directamente as diferentes dosagens de arando e as várias formulações e que avaliem segurança, tolerabilidade e duração adequadas para o tratamento profiláctico.

Ainda assim, existe um benefício potencial dos produtos de arando na profilaxia das ITU recorrentes, especialmente como alternativa para as mulheres saudáveis que preferem evitar ou que não respondem à antibioterapia profiláctica.

\section{REFERÊNCIAS BIBLIOGRÁFICAS}

1. Dwyer $P L, O$ Reilly $M$. Recurrent urinary tract infection in the female. Curr Opin Obstet Gynecol 2002 Oct; 14 (5): 537-43.

2. Guay DR. Contemporary management of uncomplicated urinary tract infections. Drugs 2008; 68 (9): 1169-205.

3. Lynch DM. Cranberry for prevention of urinary tract infections. Am Fam Physician 2004 Dec 1; 70 (11). 2175-7.

4. Stapleton A. Novel approaches to prevention of urinary tract infections. Infect Dis Clin North Am 2003 Jun; 17 (2): 457-71.

5. Ebell MH, Siwek J, Weiss BD, Woolf SH, Susman J, Ewingman B, et al. 
Strength of Recommendation Taxonomy (SORT): a patient-centered approach to grading evidence in the medical literature. Am Fam Physician 2004 Feb 1; 69 (3): 548-56.

6. Hooton T, Stamm W. Recurrent urinary tract infection in women. UpToDate 2009. Disponível em: http://www.uptodate.com/online/content/topic.do?topickey=uti_Infe/2352\&view=print [acedido em 15/12/2010].

7. Bandolier. Cranberry to prevent UTI. Bandolier 2006; May: 147-4. Disponível em: http://www.medicine.ox.ac.uk/bandolier/band147/ b147-4.html [acedido em 15/12/2010].

8. Jepson RG, Craig JC. Cranberries for preventing urinary tract infections. Cochrane Database Syst Rev 2008 Jan 23; (4): CD001321.

9. Guay DPR. Cranberry and urinary tract infections. Drugs 2009; 69 (7): 775-807.

10. McMurdo ME, Argo I, Phillips G, Daly F, Davey P. Cranberry or trimetoprim for the prevention of recurrent urinary tract infections? A randomized controled trial in older women. J Antimicrob Chemother 2009 Feb; 63 (2): 389-95.

11. Epp A, Larochelle A, Lovatsis D, Walter JE, Easton W, Farrell SA, et al. Recurrent urinary tract infection. J Obstet Gynaecol Can 2010 Nov; 32 (11): 1082-101.

12. Scottish Intercollegiate Guidelines Network. Management of suspected bacterial urinary tract infection in adults. A national clinical guide- line. Scottish Intercollegiate Guidelines Network. 2006; 88. Disponível em: http://www.sign.ac.uk/pdf/qrg88.pdf [acedido em 15/12/2010].

13. EBM Guidelines 2009. Treatment of urinary tract infections. Disponível em: http://ebmg.onlinelibrary.wiley.com/ebmg/ltk.koti [acedido em 15/12/2010].

14. Grabe M, Bjerklund-Johansen TE, Botto $H$, Çek M, Naber KG, Tenke P, et al. Guidelines on Urological Infections; 2010. Disponível em: http:// www.uroweb.org/gls/pdf/Urological\%20Infections\%202010.pdf [acedido em 15/12/2010].

\section{CONFLITOS DE INTERESSE}

Os autores declaram ausência de conflitos de interesses e financiamento do estudo.

\author{
ENDEREÇO PARA CORRESPONDÊNCIA \\ Alexandra Pina \\ R. Alfredo Cunha 365 \\ 4450-021 Matosinhos \\ E-mail: amspina@gmail.com
}

Recebido em 20/05/2011

Aceite para publicação em 28/07/2011

\section{ABSTRACT}

CRANBERRIES FOR PREVENTION OF RECURRENT URINARY TRACT INFECTIONS:AN EVIDENCE BASED REVIEW Introduction: Urinary tract infections (UTIs) are an important health problem, both in terms of morbidity and economic impact. Emerging antibiotic resistance increases the need for alternative preventive approaches.

Aim: To review the available evidence on the efficacy of cranberries in preventing recurrent uncomplicated UTIs in adult women.

Sources: MEDLINE database and the following evidence based medical sites: UPToDate, Clinical Evidence, Bandolier, EBM Online, ACP Journal Club, TRIP, InfoPOEMs, The Cochrane Library, DARE, Guidelines Finder, National Guideline Clearinghouse, Canadian Medical Association Infobase, USPSTF, NIC and Canadian Task Force on Preventive Health Care.

Methods: Search for systematic reviews, meta-analyses, clinical guidelines and randomized clinical trials published between January 2000 and December 2010 in English, Portuguese and Spanish. We used the MeSH terms: Vaccinium macrocarpon and UTI and the word cranberry. The American Family Physician `s Strength of Recommendation Taxonomy was used to establish the quality of the studies and define the strength of the recommendation.

Results: Of the 127 articles found, only nine fulfilled the inclusion criteria and were selected. These included one clinical decision support system, three systematic reviews, four clinical guidelines and one clinical trial. In its various forms, cranberries seem to effectively decrease the recurrence of UTIs in adult women without associated comorbidities

Discussion: Available evidence shows that cranberries are beneficial in preventing recurrent urinary infections (Strength of Recommendation B). However, this recommendation is limited by the heterogeneity of studies and lack of agreement concerning dosage and presentation. Better studies are needed to establish safety and tolerability as well as the adequate dosage, presentation and duration of the treatment.

Keywords: Cranberry; Urinary Tract Infection; Vaccinium macrocarpon. 